\title{
Introduction to a Major Project of the National Social Science Fund: Research on China's Industrial Heritage Conservation System
}

\author{
Subin $\mathrm{Xu}^{*}$ and Nobuo Aoki \\ School of Architecture, Tianjin University, Tianjin, China \\ * Corresponding author: 1421750993@qq.com
}

\begin{abstract}
Since the 1980s, China has undergone a series of important changes including industrial restructuring, land transfer, and urbanisation. These have given rise to the issue of how to address industrial heritage within many cities. The author's research team has undertaken a National Social Science's Major Project (the highest level of Chinese national social science research) titled 'Systematic Research on the Protection of Urban Industrial Heritage in Modern China'. This is the first nationwide and systematic study of industrial heritage in China. This paper provides a brief introduction to the study's framework and current research achievements, including: (1) the study of industrial modernisation processes, including international perspectives; (2) industrial heritage information acquisition and database setup; (3) estimation of industrial heritage value; (4) studies of the planning and design strategy of industrial heritage reutilisation; and (5) the transition from industrial heritage conservation to the development of a cultural industry.
\end{abstract}

KEYWORDS China, industrial heritage, technological history, database, value evaluation, adaptive reuse, cultural industry

Received December 7, 2017; accepted February 20, 2018.

\section{Project Background}

In 2013, the International Research Centre for the Chinese Heritage Conservation of Tianjin University successfully applied to undertake the research project 'Systematic Research on the Protection of Urban Industrial Heritage in Modern China', thus earning the official designation of a Major Project by the National Social Science Fund ${ }^{1}$. It is a systematic research study of Chinese industrial heritage sites on a national scale, scheduled to be completed in 2018 .

Since China started its historic economic reforms in 1978, Chinese industry has entered a period of highspeed development. As such, since the mid-1980s, worldwide trends in industrial restructuring have influenced some of China's more advanced cities such as Beijing and Shanghai. The industrial structure of these cities has been rapidly transformed from traditional industries to newly developed industries and the service industry. Along with the expansion of these cities, industrial estates that were once on the outskirts of these cities have now been absorbed by central urban districts.
Moreover, since the 1980s, the Chinese method for the selling and transferring of land use rights has based some of its features on the Hong Kong Land Grant System². Therefore, central city areas, as important propellers of the urban economy, have become key focus points. In contrast, the industrial estates in urban administrative centres have changed because of the continuous upgrade of industries, with former industrial areas becoming a source of contradictions and problems. The interaction between industrial restructuring and urban development has been attracting more and more attention. Because of 'differential land benefit' ${ }^{\text {' }}$, urban land renewal has been regarded as an effective approach by the local government for regional economic development. In the late 1990s, the reform of state-owned enterprises in China promoted the further upgrading of industrial estates in central urban areas. However, many industrial heritage sites that constitute potential physical resources were left idle or demolished. Since the $21^{\text {st }}$ century, many second-tier Chinese cities have been involved in this process, which means that ever-larger numbers of industrial factories 
and heritage covering lands and fields will be included in this transformation. Consequently, many industrial heritage sites have disappeared during this rapid and uninterrupted process of urban regeneration.

The disappearance of heritage sites has stimulated much research on this matter. This can be traced back to research in the 1980s concerning Chinese modern industrial heritage. After the process of national reforms and opening up to the international sphere, cooperation between China and Japan with respect to the survey of Chinese modern architecture brought new opportunities for research on Chinese modern architecture. For example, the 'Survey Report of Architecture in Dongjuzi Area', which relates to the westernisation movement analysed in the Tianjin Jianzhu Zonglan (Overview of Tianjin modern architecture, 1989), includes existing conditions and the mapping of the architecture of the Tianjin East Machinery Bureau. Research into Chinese industrial heritage subsequently reached its peak in 2009 (Aoki, Zhang and Xu 2016).

Moreover, industrial heritage protection in China is improving in terms of adopted conservation systems. Industrial heritage is gradually finding its way into the national protected heritage list, more so since the publication of the $5^{\text {th }}$ Batch of State-Protected Key Historic Sites. Moreover, industrial heritage was selected as the main theme of the International Day of Monuments and Sites in 2006, and the first Industrial Heritage Protection Meeting was held in Wuxi, in which the Wuxi Proposal was drafted. In May 2006, the State Administration of Cultural Heritage promulgated its Notice on Strengthening Industrial Heritage Protection, which officially marked the beginning of industrial heritage research and protection. After then, 18 sites of industrial heritage have been identified among the 6th Batch of State-Protected Key Historical Sites published by the State Council in $2006^{4}$. A further 84 sites were identified in 2013 in the $7^{\text {th }}$ Batch, accounting for $2 \%$ of the 4,296 State-Protected Sites. However, because of ambiguities in the scope and definition of industrial heritage, there are differences in how different professionals interpret these statistics. Despite this problem, it is clear that in comparison with other kinds of heritage, industrial heritage deserves greater attention in research and protection efforts.

In recent years, this issue has attracted growing attention from different academic societies. To date, the most relevant events have been the First Chinese Academic Forum of Industrial Heritage and the conference of the Industrial Architecture Heritage
Academic Committee (IAHAC) of the Architectural Society of China. During this conference, IAHAC raised the Beijing Proposal concerning saving Chinese industrial heritage. Since then, this conference has been held in different Chinese cities, with proceedings published every year. In 2014, the Department of Industrial Heritage was established, attached to the Historical and Cultural City Committee of the Chinese Society for Urban Studies, as well as the Industrial Heritage Committee of the Chinese Society of Cultural Relics. Moreover, the Ministry of Industry and Information Technology founded the Industrial Culture Development Centre. Hence, the establishment and work of these academic committees and organisations are promoting industrial heritage research to a new stage of interdisciplinary development.

\section{Research Directions}

Nevertheless, based on the state of the above-described research, there remain several serious deficiencies in Chinese industrial heritage research. These concern the following issues: the history of Chinese industrial technology considered from the perspective of international exchanges of knowledge; the census of industrial heritage and nationwide acquisition of information, along with research on the establishment of a database that allows for data visualisation; research on systematic value estimation of Chinese industrial heritage; conducting a comprehensive survey of the current state of heritage conservation/ regeneration, and strategic research on integrated planning, as well as the reconstruction and re-utilisation of individual buildings; and research on current survey efforts, as well as urban cultural policies, motivation analysis, and the typology of industrial heritage, which can be viewed as a form of cultural industry.

Regarding the above issues, this research project has five main subtopics: the study of industrial modernisation including international perspectives; industrial heritage information acquisition and database creation; industrial heritage value assessment; planning and design strategy studies concerning industrial heritage re-utilisation; and transition from industrial heritage conservation to the development of a cultural industry.

In terms of research methodology, several approaches were adopted: international comparative studies; integrated research of documentary sources and oral history; field surveys of pilot areas; digital information management, three-dimensional laser scanning, photography, and mapping technology; and a number of case studies were conducted. 


\section{Research Achievements: Summary}

\section{Chinese Industrial Modernisation Seen from Intra- and International Perspectives}

\section{Cross-cultural Studies}

This research focuses on the universal values of Chinese industrial heritage. On the one hand, we are concerned about the influence of industrial revolutions in Western countries. For this reason, the Kailuan Coal Mine of Tangshan was selected as a case study to analyse British influences on coal mining technology and mine construction (Xu, Hao, and Aoki 2016). On the other hand, this research is also focused on the influences arising from interactions with other Asian countries, such as the development of Tianjin's industry and commerce under Japan's influence (Xu 2014) and the travel of Korean craftsmen to China to learn about various technologies at the Tianjin Machinery Bureau (He 2009).

\section{Research on the History of Technology}

China's modern technology is developing under interactions between Chinese tradition and Western influences and contains some important objects of research including construction technology and equipment technology. In terms of traditional construction techniques, we can refer to the construction plans of the Tianjin East Machinery Bureau and West Machinery Bureau at the Qing Dynasty Yangshilei Archives, which have been recommended for inclusion in UNESCO's Memory of the World Register. These materials reveal the relationship between traditional architectural design and modern industrial facilities (Ji, Xu, and Aoki 2011), in which there is a strong influence from Western countries. As a whole, this research considers long-span construction technologies (Lai 2016), brickmaking techniques (Lai 2016; Lai and Xu 2017; Yu, Qian, and Li 2017), and cement technology (Lai, 2016), as well as research on the production lines of different industries (Yu, Aoki, and Xu 2016).

\section{Research on the History of Heritage Integrity}

There are two important issues facing industrial heritage in China: appropriate methodological approaches towards authenticity and integrity protection. Since the introduction of land transfer policy, land resources have become one of the most important fiscal revenue sources of national and local government entities. Consequently, it is crucial to consider the boundaries and definitions of the integrity of heritage. To this end, three types of integrity have been researched: integrity of industry networks; the integrity of factories; and the integrity of production lines (equipment included). Based on these, basic conservation principles and grades of value assessment according to various types of heritage can be formulated to minimise the loss of heritage due to land shortage (Xu and Aoki 2012).

As there are different production lines for different industries, specific methods of value assessment are essential. This research examines the integrity of infrastructure as well as the specificity of production lines in different industries such as the manufacturing industry, transportation, and mining industry. It also proposes a number of basic principles for conservation (Yu, Aoki, and $\mathrm{Xu}$ 2016). Moreover, the case studies are also considered in accordance with other types of industrial heritage from different time periods (Sun, Xu, and Aoki 2015).

\section{Industrial Heritage Information Acquisition and Database Creation}

Temporal Evolution of Chinese Modern Industry Because of limitations in research methods, previous studies have mainly been based on the documents and personal opinions of researchers. In contrast, this research is a comprehensive multi-perspective study, in which geographic information system software (GIS) has been utilised for the assessment and reconfirmation of the historical stages of industrial heritage ${ }^{5}$. Using this method, data from nearly 2,200 related industrial factories in modern China have been collected, including specific establishment dates for 2,131 of them. Because of the sharply increasing number of factories since the foundation of the People's Republic of China, and the near-lack of varying opinions about their temporal development, this research focuses on factories built between 1840 and 1949 as the main object of study (Figure 1).

\section{Establishment of a Historical Database}

As mentioned above, this research considers industrial factories in modern China established during 1840-1949 as its research objects; these were selected from previous studies that featured relatively complete information and displayed a systematic approach towards careful documentation and data collection, as well as a high number of citations ${ }^{6}$. With regard to the factories and plants possessing specific set-up times and locations, their information was recorded in the GIS database as a point data set, with positional accuracy controls carried out within the range of the county (district) of the factories. The name, set-up time, industry type, and the capital type (such as state-owned enterprises, private enterprises) of each factory were also collected to establish a historical 


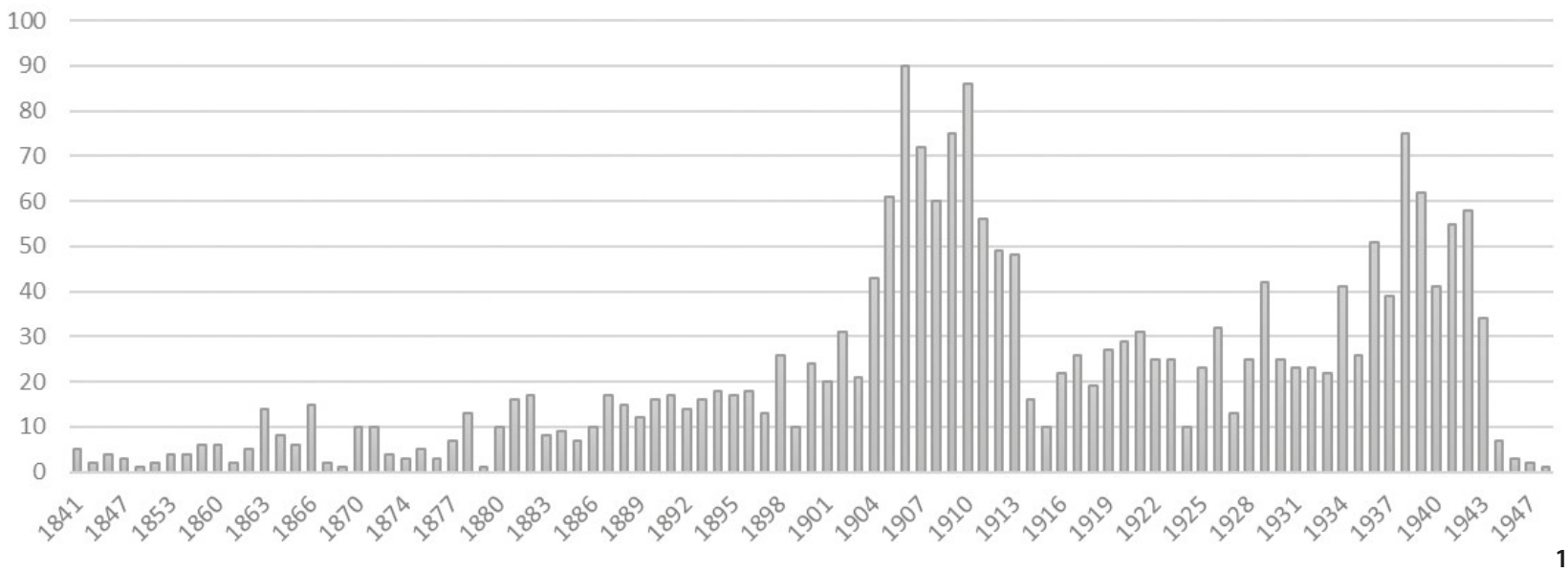

Figure 1 Statistical distribution of Chinese modern factories during the period 1840-1948 (Source: Subin Xu).

and geographic information database of Chinese modern industry. For railway data, the research team collected polyline datasets including attribute information such as name, establishment date, operation time, and number of construction workers (Liu, 2017).

The base map was adapted from the CHGIS system (V5 version, 1911) (CHGIS, Version: 5 (c) Fairbank Centre for Chinese Studies and the Institute for Chinese Historical Geography at Fudan University, 2012.) developed by the Fairbank Centre for China Studies, Harvard University and the Centre for Historical Geographical Studies of Fudan University. The basic unit for spatial analysis is county-level administrations (excluding Taiwan, China), and where counties lacked an administrative boundary, the municipal administrative boundaries were adopted instead. This research is founded upon the basic assumption that the base data is in itself reliable. The collected information includes many modern factories and mines in modern China as typical examples that can be representative of the distribution characteristics of the research period. The second assumption is that the administrative boundaries were relatively stable over the period of this dataset.

The resulting maps showcasing the relative density of factories demonstrate the temporal-spatial evolution of modern industry in China and mines during different periods (Figure 2a-2g).

\section{Establishment of the Current Database}

Procedural standardisation was considered to be an essential precondition for the data collection work. Therefore, an integrated framework was established before developing the database. The following represents the basic procedure for the data collection of industrial heritage facilities:
1. Establish a standard set of regulations and plans: compile a unified 'Census Form of Chinese Industrial Heritages', and create the necessary regulations and time schedule;

2. Collection of information on the census area: compile data from the Third National Archaeological Survey of relevant areas, as well as local documentation of modern industrial facilities, CAD drawings, aerial photos, and site plans; and

3. Implementation of the field survey: based on the information collected from archival sources it became possible for both professionals and volunteers to conduct the field survey with the cooperation of related governmental departments.

Concerning the status of Chinese industrial heritage research conducted up until July 2017, we can account for 1,487 industrial heritage sites in total, which include 1,391 modern industrial heritage sites and 265 sites of reconstruction and re-utilisation. The database contains information on each site, including names, establishment times, locations, addresses, degree of protection, type of functional transformation, and positional coordinates. Figure 3 shows the number of industrial heritage sites in various Chinese cities. The top five cities with the largest number of industrial heritage sites are Shanghai municipality, Guangdong Province, Jiangsu Province, Liaoning Province, and Tianjin.

During the process of creating the database, three database levels were implemented. First, there is the national level database, which is hoped to become an important reference in the future as the first national survey of its kind. Second, the database at the city level focuses on different industries and their distributions, as well as the usage condition of key industrial cities 

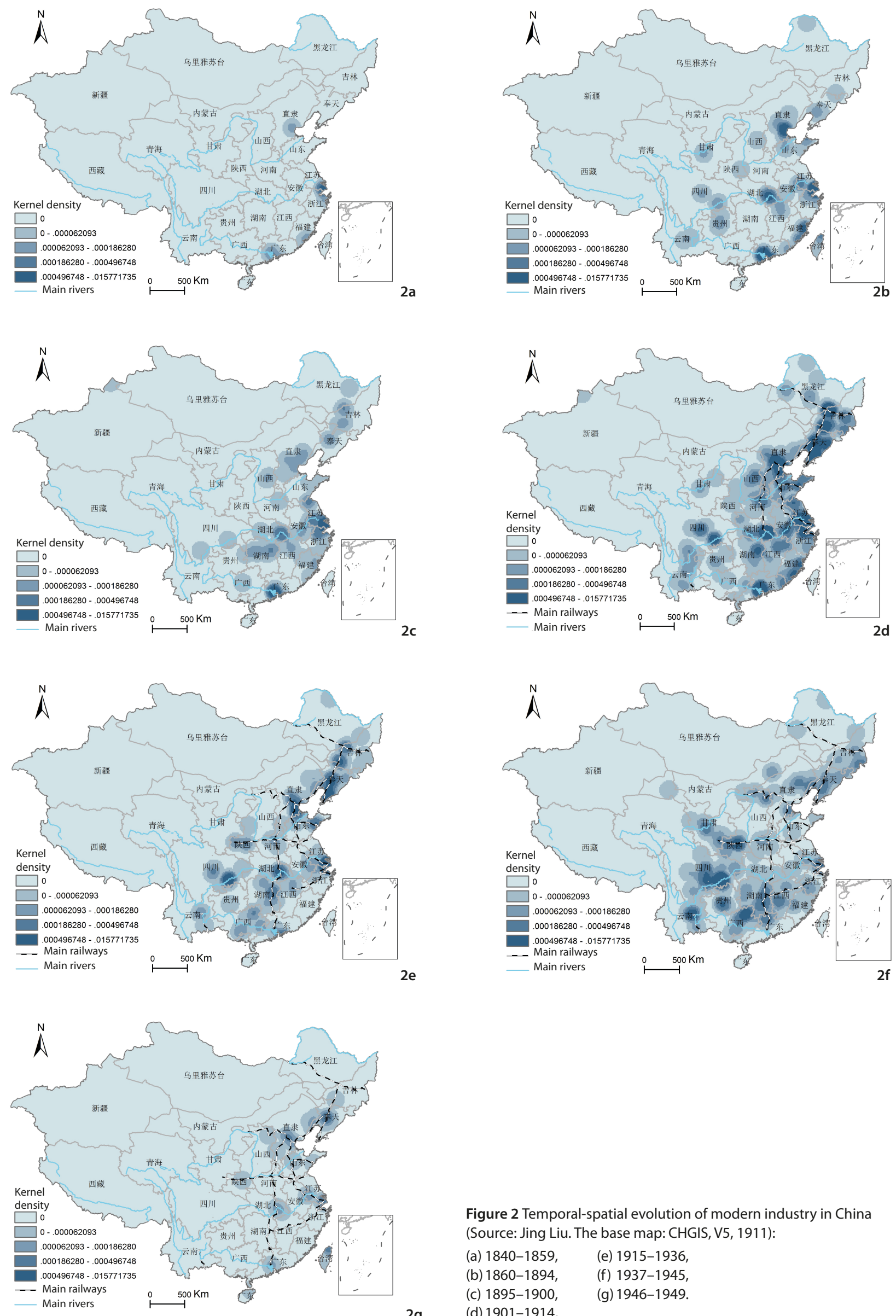

Figure 2 Temporal-spatial evolution of modern industry in China (Source: Jing Liu. The base map: CHGIS, V5, 1911):
(a) 1840-1859,
(e) 1915-1936,
(b) 1860-1894,
(f) $1937-1945$
(c) $1895-1900$,
(g) 1946-1949. 


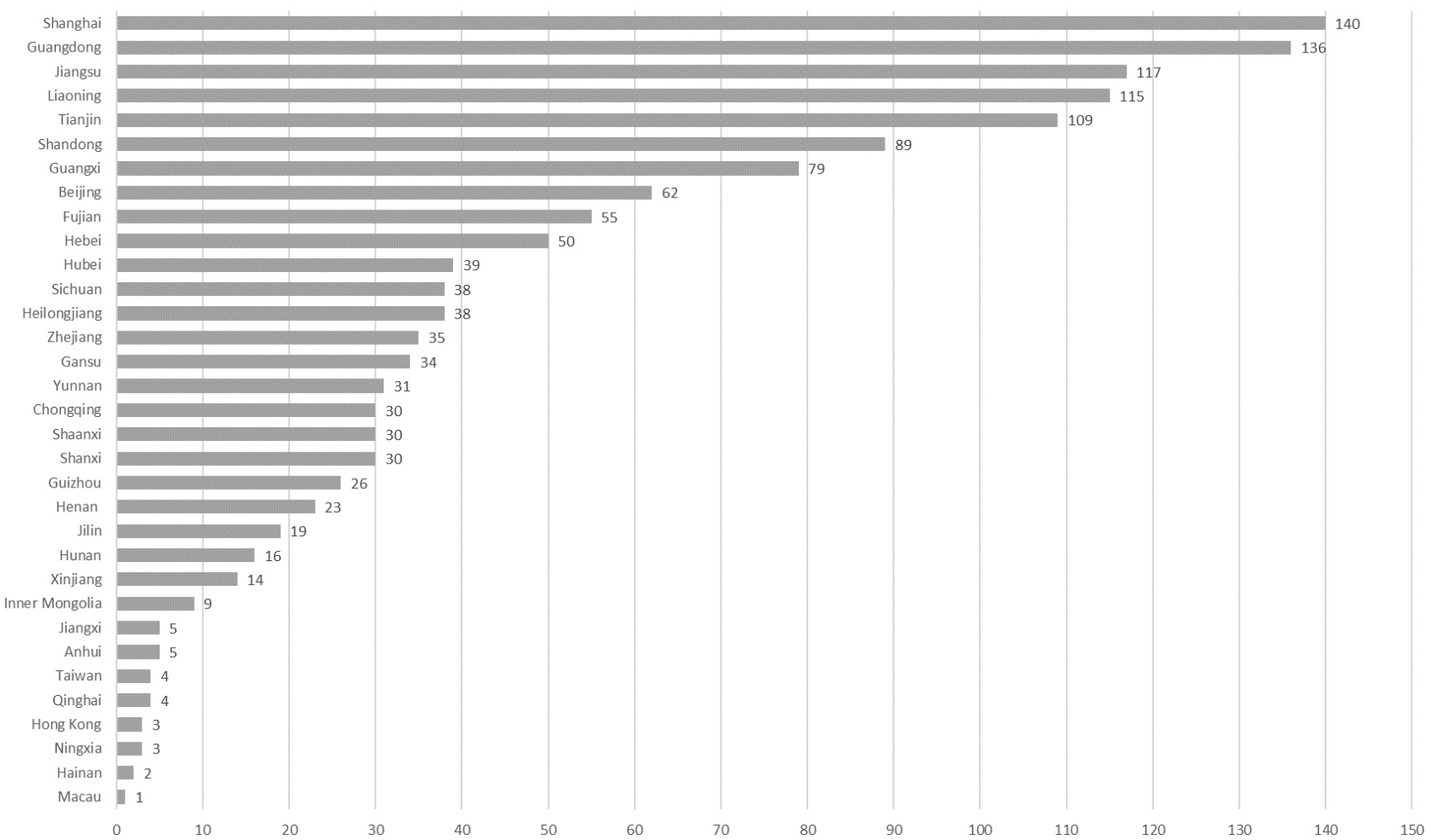

Figure 3 The database of Chinese industrial heritage sites contains 1487 industrial heritage sites in total (up to July 2017), which includes 1391 modern industrial heritage sites and 265 sites of reconstruction and reutilization (Source: Jiahao Zhang).

in which Tianjin is regarded as a case study. The third level is that of Key Cultural Relics Protection Units. This research can be considered a reference for formulating conservation plans for cultural relics to further strengthen their management. This research takes the North Navy's Taku Dockyard of Tianjin as an example. The third level includes information on multiple aspects of the facilities, from their historical status up to their current status, as well as architectural and environmental information. The concept of temporal data has also been adopted to allow for queries with time variables (Zhang, $\mathrm{Xu}$, and Aoki 2016).

Moreover, the BIM model has been adopted in the database for recording information about the facilities' architecture and equipment, which, when connected to the GIS database, can provide abundant and precise information (Du 2013; Shi 2014).

\section{Industrial Heritage Value Assessment}

General Framework for Industrial Heritage Value Assessment

Until now, the main difficulties arising during the process of industrial heritage evaluation were fragmented/unconsolidated theories, excessive quantification, and a lack of feasibility. In response to these issues, our research team reviewed and reconstructed a theoretical approach towards heritage evaluation.

Following a traditional view of economics, there is physical capital (factories and equipment), human capital (workers), and natural capital (mines). These forms of capital and their value can all be found in industrial facilities. Moreover, they include cultural capital to some extent, as some of the industrial facilities have witnessed important historical moments and became heritage sites, as discussed by David Throsby in 'Economics and Culture' (Figure 4). As time goes by, the first three kinds of capital tend to degrade, while cultural capital may appreciate in value. Furthermore, these four types of capital can be evaluated using economic assessment methods.

If the framework of industrial heritage value can be metaphorised as a 'duck egg', its 'yolk' could represent the 'intrinsic value', which is the original value of industrial heritage. If the total intrinsic value of an industrial heritage site is A, its physical capital is Ap, its human capital is Ah, its natural capital is An, and its cultural capital is Ac, then $A=A p+A h+A n+A c$. The intrinsic value comprises the main core of industrial heritage, which should be evaluated and graded.

However, for some industrial heritage sites, there is a need to reconstruct and regenerate; if so, we define the 


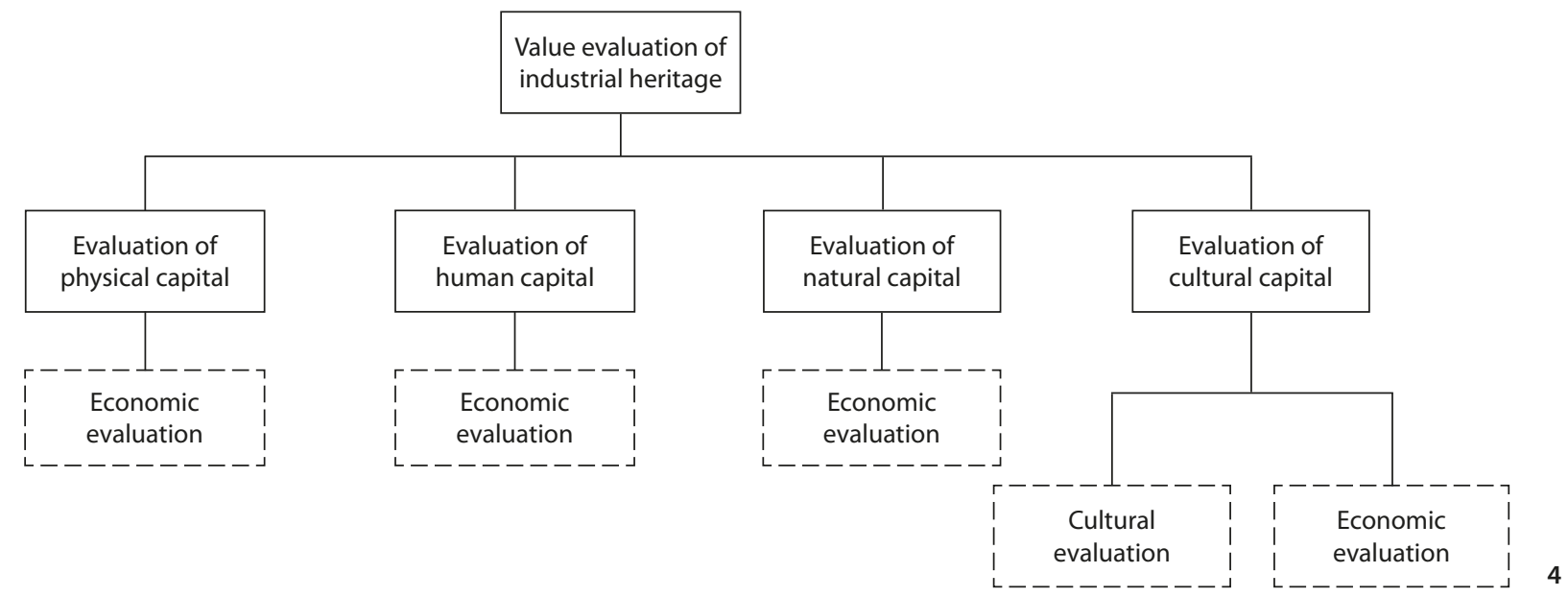

Figure 4 Classification of value evaluation of industrial heritage (Source: Subin Xu).

value added after the regeneration process as the 'creative value', which is represented by the metaphor of a 'egg white' and symbolised as B. Therefore, the physical capital of the creative value (such as newly built factories) is $\mathrm{Bp}$, human capital (new service industries) is Bh, natural capital (new landscape and vistas) is Bn, and cultural capital is $\mathrm{Bc}$ (painting, literary or animation creation in the industrial heritage site as a new creative industry). Thus, the total creative value will be $\mathrm{B}=\mathrm{Bp}+\mathrm{Bh}+\mathrm{Bn}+$ Bc (Figure 5).

According to David Throsby's concentric model, there are four layers of cultural industry, starting from the centre to the outside. The first layer is the core creative arts including literature, music, performance arts and visual arts, while the second layer includes other core cultural industries represented by film, museums, art galleries and libraries. The third layer relates to wider cultural industries including heritage services, publishing and printing media, sound recording, television and radio, video, and computer games. The outermost layer is related industries, which include advertising, architecture, design, and fashion (Throsby 2010). Their overall assumed level of creativity decreases from the inside out.

Moreover, the 'eggshell' refers to positive spillover effects, which are defined as an unintentional or uncompensated benefit or cost, flowing to another economic agent. For example, a museum in a downtown area could create employment, revenue, and other economic opportunities for enterprises and residents nearby. These effects may be important in the local or regional economic evaluation, but are also difficult to properly quantify (Throsby 2001, Throsby 2010). For instance, a given cultural heritage can drive the development of surrounding transportation, commercial, and hotel facilities.
Therefore, we propose the term of 'cultural magnetism' which is driven by the intrinsic value and creative value. Only if the 'cultural magnetism' of a cultural heritage is well protected can its sustainable development be guaranteed (Xu and Aoki 2017).

\section{Evaluation of Cultural Capital}

The research team also further explored the value of cultural capital. According to the Law of the People's Republic of China on the Protection of Cultural Relics, this emphasises the historical, artistic, and scientific value of heritage. Besides these, the Principles for the Conservation of Heritage Sites in China mentions the type of social and cultural values. Because there is a wide range of heritage types with their respective attributes and characteristics, there should be a secondary standard based on their historical, artistic, scientific, social, and cultural value. In this regard, the guidelines in Britain could be an appropriate point of reference. There are different specific guidelines according to the different types of heritage in the United Kingdom, including industrial heritage. While referring to UK guidelines as well as the opinion of Chinese experts ${ }^{7}$, the research team proposed the 'Value Evaluation Guideline of Chinese Industrial Heritage' (trial edition). It includes 12 criteria used for assessing the value of industrial heritage as follows: age; historical importance; industrial equipment and technology; architectural design and construction technology; cultural and affective identity; promoting the development of local society; conditions of preservation, repair, or reconstruction; integrity of regional industry chain; representativeness and rareness; vulnerability; conditions of documentary records; and potential value. Furthermore, value assessment 


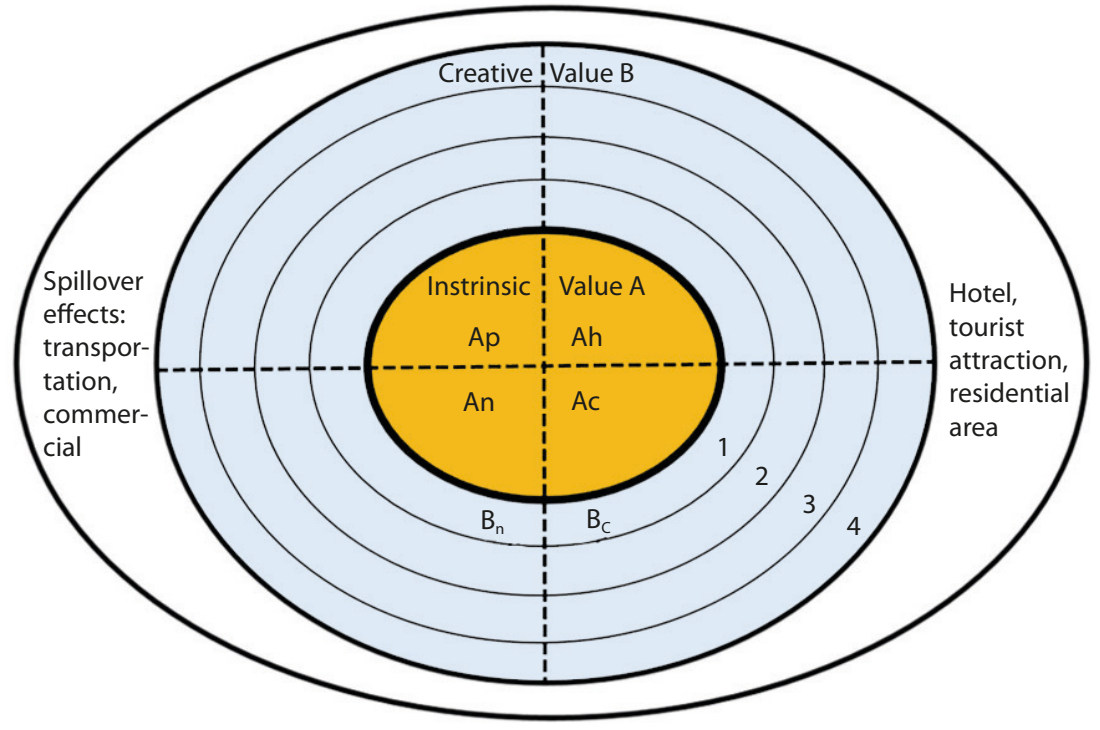

Figure 5 Framework of the value of industrial 5 heritage (Source: Subin Xu). approaches for different industries were explored in detail as guidelines for practical research and protection actions (Yu, Aoki, and Xu 2016). The negative value of industrial heritage has also been taken into consideration (Aoki, Xu, and Wang 2016).

\section{Calculation Approaches towards Cultural Value As- sessment}

In this context, a specific approach to the calculation of cultural value can be considered. In April 2017, our research team finished a questionnaire survey at the Beijing 798 Art Zone and collected 303 valid questionnaires. Based on the economic assessment principles espoused by the Travel Cost Method, cultural value is the summation of the overall travel cost (transportation, accommodation, and food, admission, entertainment, souvenirs, etc.), opportunity cost of the salary lost during the total travel time, and consumer surplus (the difference between the price the consumer is willing to pay and the actual price they pay for it), which is 1,472.2949 million CNY. Conversely, the total cultural value measured by the Contingent Valuation Method is 49,578.6166 million CNY. Thus, it can be demonstrated that the administrative department of the 798 Art Zone should enhance its service quality and improve visitor satisfaction. Additionally, the cultural heritage of the art zone should be protected to improve its cultural atmosphere and attract more visitors (Chen and Xu 2017).

\section{Strategies for Reconstruction and Regeneration}

\section{Field Survey}

This research selected 185 sites of industrial heritage (and national mine parks) that have been reconstructed and regenerated for analysis, with the data sources mainly being CNKI, Ministry of Land and Resources, State Administration of Cultural Heritage, and the websites of national and local government entities. Subsequently, 156 regenerated and transformed industrial heritage sites were selected for the field survey. Among them, 71 sites were located in eastern China, which accounted for $45.5 \%$ percent of the sample; 44 sites were located in northern China (28.21\%); and $17.95 \%$ were located in the central-southern area of China (with an increase in the number of sites in recent years). As for the northeast, southwest and northwest areas, they accounted for just $3.21 \%, 3.85 \%$, and $1.28 \%$, respectively.

Based on the field survey, the types of transformation of industrial heritage sites mainly include those of cultural industry, museums, urban public space, business space, offices and residential developments. Among these, cultural industry occupies the largest proportion with $49.68 \%$; museum, business space and office space occupy the following positions with $9.62 \%, 9.94 \%$ and $12.35 \%$ respectively, totalling $31.89 \%$. Urban public spaces, residential development, and cultural facilities transformations account for a much smaller proportion, at only $13.15 \%$. 


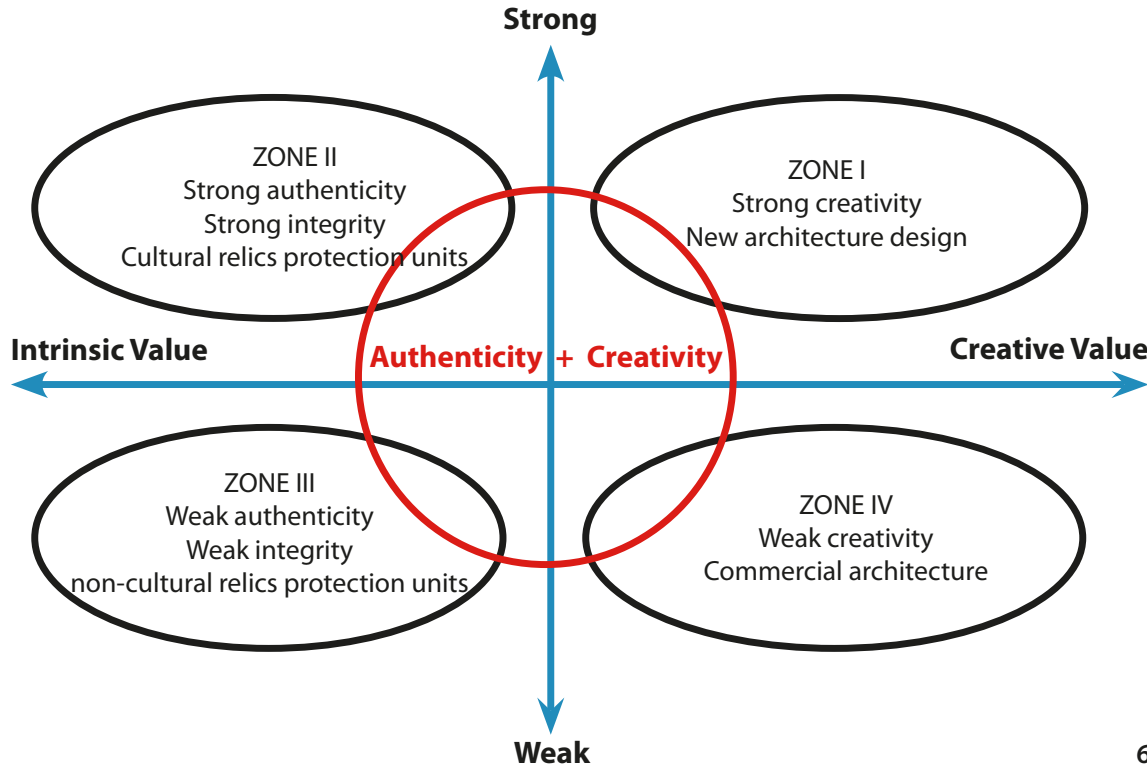

Figure 6 Schematic diagram of the ideal aims of heritage reconstruction 6 and regeneration (Source: Subin $\mathrm{Xu}$ ).

\section{Dual Principle of Authenticity/Creativity and Case Studies}

For institutional reasons, projects of industrial heritage conservation are isolated from wider regeneration efforts in China. However, almost all heritage sites need to be evaluated in terms of either a formulation of conservation plans or architectural design plans, according to the Guidelines for the Formulation of Conservation Planning for Key Cultural Relic Protection Units of China. Other unlisted industrial heritage sites are mainly designed and transformed by architects. According to the survey on 50 reconstructed industrial heritage sites, only eight were evaluated before reconstruction work occurred (Hao, Xue, Chen, and Du 2012).

To properly address the issue of placing undue emphasis on 'museum-type' conservation projects or to avoid focusing more on the subjective feeling of designers rather than assessing the value of industrial heritage facilities, this study follows the dual principle of authenticity and creativity. Concerning authenticity, it enables us to discuss approaches towards maintaining the authenticity of heritage sites based on the aspects of spatial design, façade design, structural system design, construction design, and equipment design, among others (Feng 2016). Regarding creativity, the research explored several creative approaches for industrial heritage transformation, including external space reconstruction (e.g., regeneration and conservation of the façade, restoration of the roof, and the extension of the architectural façade), and interior space reconstruction (e.g., the function and layout, reinstallation of the interior space of architecture, and the restoration of the interior spaces) (Liu 2016).

\section{Reconstruction and Regeneration Strategy}

The aims of heritage reconstruction and regeneration are to guarantee its authenticity as well as to enable design creativity. Figure 6 depicts an ideal model of heritage reconstruction and regeneration. According to the current situation in China, it demonstrates that to achieve the proper balance of authenticity and creativity, it is best to avoid typical museum-style protection attitudes with the mere consideration of intrinsic value, and conversely to avoid excessive creative design without taking authenticity and integrity into account.

In the case of ZONE I (Figure 6), the intrinsic value of the original building does not lie specifically with its status as designated heritage, and highlighting the features of the new building is more common. The Jinling Art Museum of Nanjing is a typical example. It was originally the Nanjing Colour Weaving Factory, built in the $20^{\text {th }}$ century in the 1960s and 1970s, and is located south of Nanjing, a district renowned for its history. Because the factory itself is not a cultural relic, the architect made radical transformations to its form in 2011, giving it a stronger visual impact (Figure 7).

In the case of ZONE II (Figure 6), the building's status as designated heritage becomes the main target for protection, and stricter efforts are expended to safeguard its authenticity. Within the studied examples of the transformation of industrial heritage, the protection of the west wall of the Warehouse of Four Banks in Shanghai 

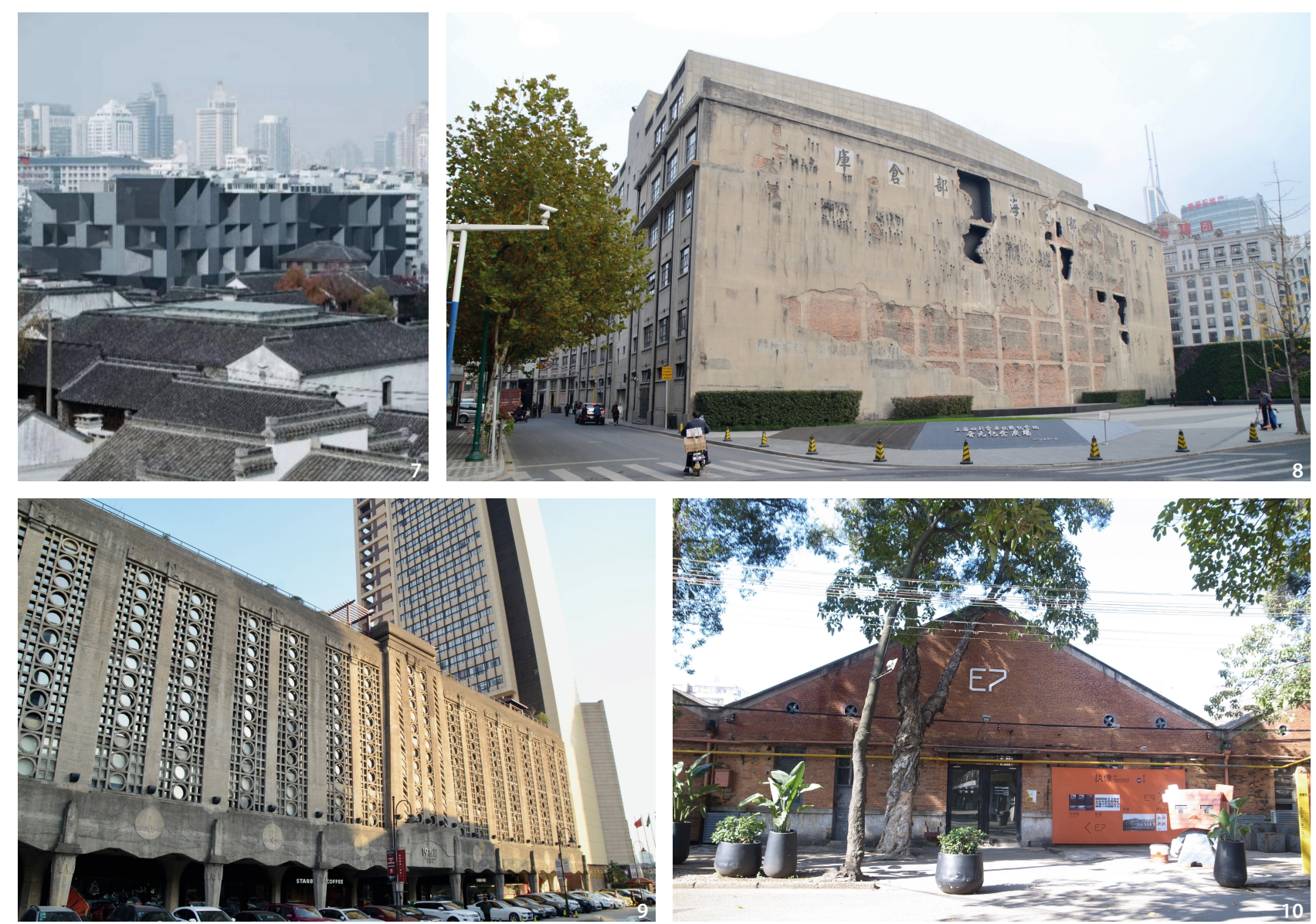

Figure 7 Jinling Art Museum in Nanjing, designed by Liu Kecheng, 2011 (Source: UED).

Figure 8 The west wall of the Warehouse of Four Banks in Shanghai, designed by Guoliang Liu etc. 2014 (Source: Zijie Zhao).

Figure 9 Old Millfun in Shanghai, Designed by Chongxin Zhao etc., 2007 (Source: Zijie Zhao).

Figure 10 Redtory Culture Industrial Park in Guangzhou, 2009 (Source: Subin Xu).

approaches the extremes of authenticity protection. The Warehouse of Four Banks was jointly built in 1931 by the Jin Cheng Bank, Central and Southern Bank, Continental Bank, and the Salt Bank. In 1937, there was continuous resistance against the advances of the Japanese army for four days and nights, resulting in several bullet holes in the west wall of the warehouse. The warehouse was designated as an outstanding historical building in Shanghai in 1994. It was transformed into a Creative Industrial Park in 2003 and classified as a cultural relics protection unit in 2014. To commemorate the resistance efforts against the Japanese army, the authenticity of the west wall of the warehouse was completely preserved in the reform design (Figure 8).

The 1933 Old Millfun in Shanghai is also a good example of how to respectfully maintain the authenticity of the original building. The original building is a slaughterhouse, and it is representative of early reinforced concrete buildings in Shanghai. The concrete structure and diversified spaces are its outstanding features. The transformation work basically respected the intrinsic value of the original building. The building is used for business activities and houses various eateries (Figure 9).

At present, most of the cultural industrial parks in China can be ascribed to ZONE III (Figure 6). Because the focus of the cultural and creative industrial parks is to drive the local economy rather than emphasise the creative design of its architecture, most were designed by the artists who rented the building rather than architects. For example, Redtory in Guangzhou, previously a canning factory, is now used as office space but lacks a creative building design. Another reason for this insufficiency in its creative design is that local property policies are unstable (Figure 10).

Industrial buildings devoid of intrinsic value belong in ZONE IV (Figure 6). However, there is a large number of industrial buildings that have not yet been exploited; they may hold potential value for development, once a proper assessment is conducted. A significant transformation may take place after a well-considered transformation design is implemented. 

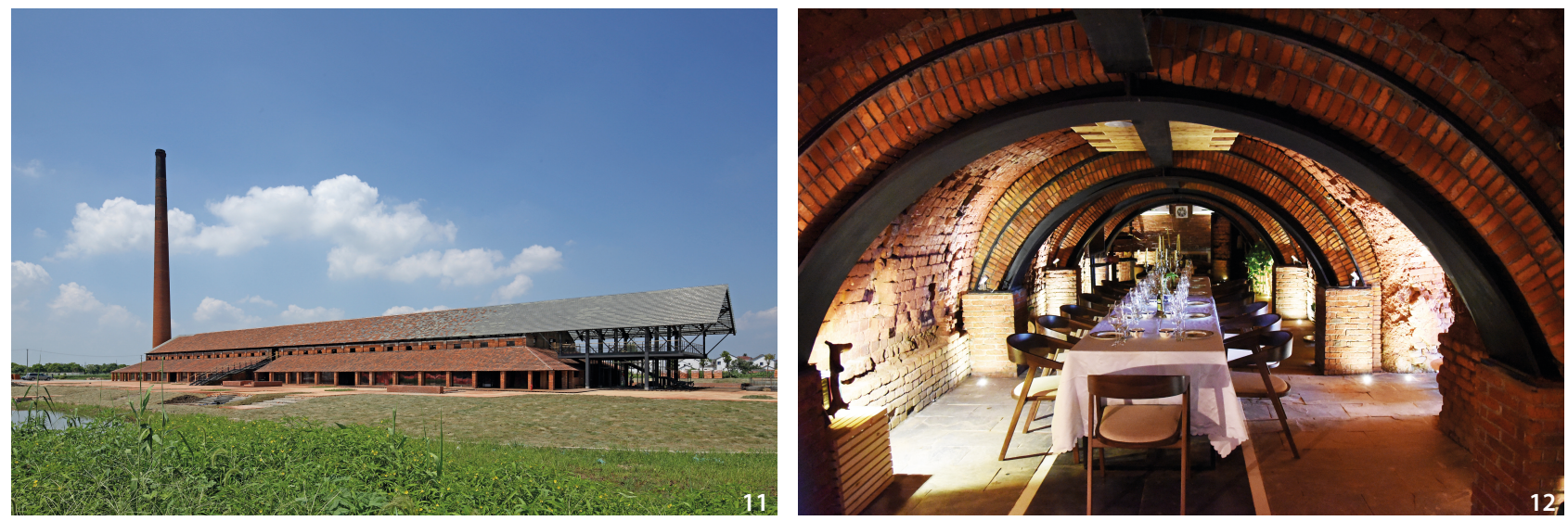

Figure 11 The reconstructed brick and tile factory, designed by Kai Cui Land-Based Rationalism D-R-C, 2016 (Source: Guangyuan Zhang). Figure 12 Brick and tile factory transformed into a dining hall, designed by Kai Cui Land-Based Rationalism D-R-C, 2016 (Source: Haian Guo). Figure 13 Steel stairs leading to the ancient kiln Museum in the second floor, designed by Kai Cui Local Design and Research Centre, 2016 (Source: Guangyuan Zhang).

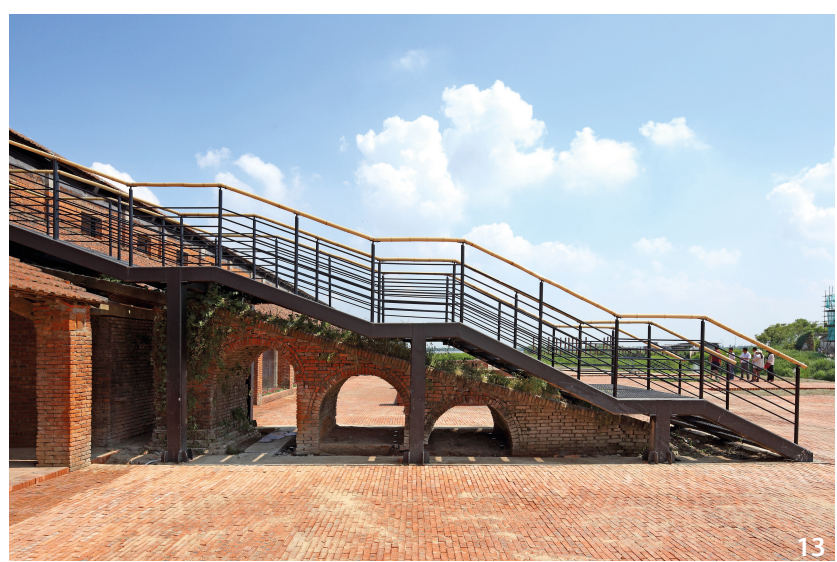

The balance of authenticity and creativity is the key to the adaptive reuse design of industrial heritage. The brick tile factory in the town of Jinxi, Jiangsu Province stands as a meaningful example of protection schemes concerned with both authenticity and creativity. The factory once produced 'gold brick' - a kind of high-quality brick for the Forbidden City. The factory used a German Hoffman kiln in modern times and was abandoned in the 1980s. At present, it represents the intangible cultural heritage of Jiangsu Province. In the spirit of respecting its heritage, the designer protected the integrity of the Hoffman kiln and chimney. The first story serves as a restaurant, and the second as an Ancient Kiln Museum. To strengthen the original structure, the designer interspersed a steel structure throughout the old building's roof and built a steel staircase covering the original brick stairs. The new features were inserted into the old factory. The resulting new structure strives to achieve a minimum level of intervention. This design not only protects the original brick structure, but also showcases a modern sensibility (Figures 11-13).

How does the architect ensure the authenticity of the design? This research references the 'The Sourcebook of Architecture' (third edition, first draft), which supplements the procedure of heritage conservation in the 8 th

volume of the sourcebook with the design process of historical architecture. Based on this, the chart in Figure 14 is the result of the author's revision, which proposes a general procedure for the reconstruction and regeneration of historical architecture.

From this flowchart, it is clear that the value assessment process should be a priority to avoid damage to any heritage value while having the maximum possible creative design - this represents the best state of adaptive reuse. It suggests that the value assessment process should be further supplemented during the review procedure by the administration in charge of monitoring the facility.

\section{From Industrial Heritage Conservation to the Development of a Cultural Industry}

\section{Comprehensive Survey of Present Situations/ Conditions}

The cultural industry is a new industry that has developed rapidly in recent years. This research focuses on how to address industrial heritage within the domain of cultural industry towards achieving a more sustainable design. It selected eight cities for a field survey (Beijing, Shanghai, Guangzhou, Tianjin, Chongqing, Qingdao, Xi'an, and 


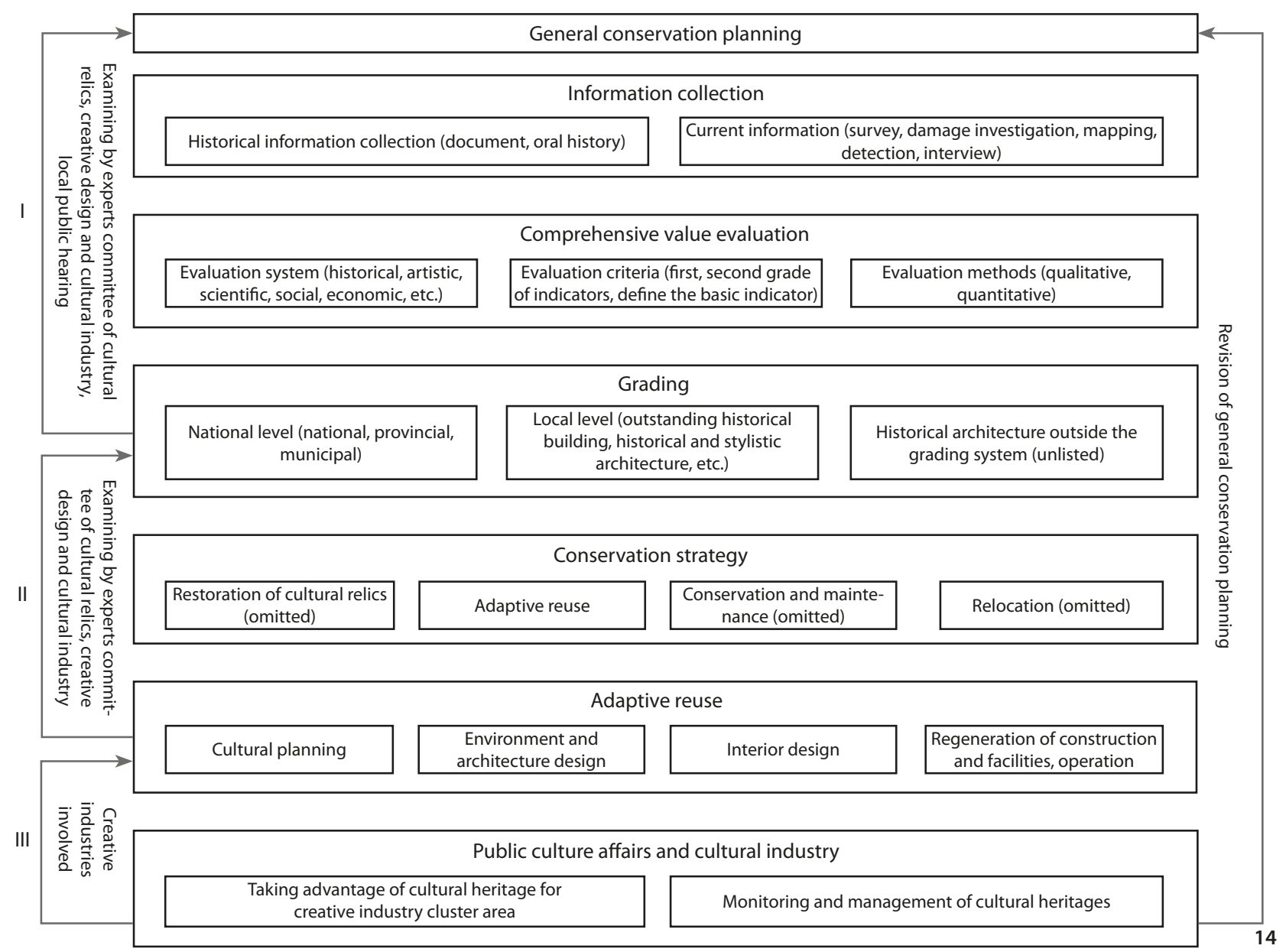

Figure 14 Procedures for historical architecture reconstruction and regeneration (Source: Subin Xu).

Fuzhou), and established a database of 190 cases. It also investigated and analysed some key cases in a number of cities including Beijing, Tianjin, Qingdao, Shanghai, Wuhan, Xian, and Guangzhou (Zhong 2016).

\section{Motivation Analysis of Regenerated Industrial Heritage as a form of Cultural Industry}

There are mainly three types of development promotion available for industrial heritage reused as a form of cultural industry in China: the allocation of land use rights, transformation and upgrading of traditional industry, and market discipline. This research explains the influence of the allocation of land use rights using case studies from Beijing and Shanghai, and resorts to the 'ShanghaiQingdao-Tianjin' pattern found in the Chinese textile industry to interpret the influences derived from the transformation and upgrading of traditional industries. It also explains the influences of market discipline in the case study of Qingdao (Zhong 2016).

\section{Main Points Regarding the Developmental Modes of Regeneration: From Industrial Heritage to Cultural Industry}

Because the modes of development and the functioning of institutions in China are different to those of Western countries, this research project, in its ultimate conclusion, takes into consideration the development modes of Chinese industrial heritage as a cultural industry with concern to the particular context of China. These modes are basically driven by universities, promotion initiatives by government bodies and property developers, and companies engaged in cultural industry activities, and each mode was analysed.

Based on these distinctions in value preferences, and the role played by dominant institutions, the actual performance and predominance of the values of transformation/ heritage differ as a result. For example, in terms of regeneration projects led by the government, they are mainly dominated by reconversion as museums and landscape parks, which become a part of the urban infrastructure for 
public service. In contrast, the value of heritage could be better embodied through closer cooperation between the government and academic research teams. In such cases, it can be said that the value of the transformation of use is not usually as well developed as a business development approach. Additionally, projects led by property developers always involve high degrees of transformation, but also less accurate interpretations of heritage value, which require the participation of an academic research team to guarantee their success (Zhong 2016). Moreover, the research team selected the Tianjin Third Cotton Textile Factory as a case study, and interpreted different development modes in the same project to showcase the flexibility of its current development mode (Qiu, Zuo, and Huang 2016). Based on the above research and achievements of the field survey, policy suggestions can be put forward.

\section{Conclusion}

This project researched and investigated Chinese industrial heritage from an international perspective, having the following characteristics:

1. Wide-range research on spatial and temporal factors. There has been no national industrial heritage census in China until now, which admittedly made the project's realisation more difficult. Our research team investigated approximately 1,400 industrial heritage sites in 33 provinces, cities, and municipalities, and visualised this complex information through a GIS database.

2. Systematic research. The integrated project was divided into five continuous and interrelated subtopics. It focused on the comprehensive and interdisciplinary study of industrial heritage with due consideration for technology history, information collection, value assessment, adaptive reuse, and cultural industry.

3. Site surveys. This project sought to provide a detailed field investigation report, and compiled site surveys, interviews, questionnaires, mapping data, and more to establish a solid foundation of comprehensive information on Chinese industrial heritage. The problems and issues discovered during the process of directly dealing with these industrial sites helped with the analysis, which further led to the proposal of the dual principles of authenticity and creativity.

4. Theoretical research. It proposes a comprehensive value assessment framework for Chinese industrial heritage featuring the involvement of physical, human and natural capital. In addition, the differences between intrinsic value and creative value were also discussed The evaluation system not only includes the typical assessment of cultural impact, but also an assessment of the site's economic impact, for which the 798 Art

Zone is a representative case study.

Finally, concerning the future prospects of this research project at the national level, it would be worthwhile to involve the input of research studies on brownfield lands containing industrial heritage. While this is a social science project with limitations in terms of research budget and the number of researchers, the careful integration of an environmental perspective could prove to be crucial for the comprehensive well-being of the future inhabitants of these sites.

\section{Notes}

1. Project number: 12\&ZD230. Chair: Subin Xu; Co-operators: Nobuo Aoki, Cong Wu, Yuru Wang, Lin Wang. Research members include staffs, doctoral and master students of International Research Centre for Chinese Cultural Heritage Conservation, School of Architecture, Tianjin University.

2. Paragraph IV, Article 10, Constitution of the People's Republic of China, revised since 1988. 'No organisation or individual may appropriate, buy, sell or otherwise engage in the transfer of land by unlawful means. The right to the use of land may be transferred according to law.

3. Differential land benefit means the different benefits relating to different grades of land, which is one of the standards of benchmark land prices.

4. Along with the old ruins of iron-making and copper mine sites, ruins of mercury processing sites, ancient kiln, ancient liquor brewhouses and shipyards, we can find in the list some notable modern industrial heritage sites such as: the Huangya cave arsenal, the Middle East railway complex, the early buildings of the Tsingtao Brewery, the Hanyeping Coal-Iron Industrial Site, Shilongba Hydropower Station, Gejiu Railway Station, Qiantang River Bridge, the former launch site of missiles and satellites known as Jiuquan Satellite Launch Center, Dashen Cotton Mill of Nantong, etc. Besides these, the first oil well of Daqing and the former site of nuclear weapons research in Qinghai were also included in the early stages of the protected sites list.

5. The data on the establishment dates of modern industrial factories was extracted mainly from Zhongguo jindai gongyeshi ziliao [Document for the Modern Industrial History of China] compiled by Zhen Chen, Luo Yao; Zhongguo jindai gongyeshi ziliao [Document for the Modern Industrial History of China (Issue 1)] 
compiled by Yutang Sun; Zhongguo jindai gongyeshi ziliao [Document for the Modern Industrial History of China (Issue 2)] compiled by Jingyu Wang.

6. The most-often consulted sources of documentation were: Zhongguo jindai gongyeshi [History of the Modern Industry of China] compiled by Cishou Zhu; Zhongguo jindai gongye fazhanshi (1840-1927) [Development History of Chinese Modern Industry (1840-1927)] compiled by Xicheng Fan and Baozhen Lu; Zhongguo jindai gongyeshi ziliao [Document for the Modern Industrial History of China (Issue 1-4)] compiled by Zhen Chen, Luo Yao; Zhongguo jindai gongyeshi ziliao [Document for the Modern Industrial History of China (Issue 1, 1840-1895)] compiled by Yutang Sun, 'Studies of Chinese Modern Economic History' by the Institute of Economics, Chinese Academy of Sciences; Zhongguo jindai gongyeshi ziliao [Document for the Modern Industrial History of China (Issue 2, 1895-1914)] compiled by Jingyu Wang; 'History of Modern Chinese Railways' compiled by Yonggang Yang, etc.

7. In terms of the selection of evaluation criteria, our research team distributed a questionnaire survey and engaged in discussions with participating experts at the third Chinese Academic Forum of Industrial Heritage in 2012. Based on the responses, five preliminary criteria were proposed and ranked. As the research progressed, a second questionnaire survey was presented at the fourth Chinese Academic Forum of Industrial Heritage. At that time, we proposed four basic values and eight factors of influence as first-grade indicators, which were further divided into secondary indicators. Experts were also asked to attribute indexing weights. During the meeting, the experts enthusiastically discussed the proposed indicators and gave further advice and suggestions. In May 2014, based on the two surveys, the research team proposed 12 criteria, which became a source of consensus at the founding conference of the Industrial Heritage Committee subordinated to the Chinese Society of Cultural Relics. The condensed 12 criteria are comprehensive, systematic, and feasible, embodying the representative characteristics of industrial heritages and received good appraisals by the participating experts. At the same time, another questionnaire survey was distributed within the national academic field to approximately 200 professionals. The more than 50 valid questionnaires collected show that the experts mostly agreed with the value assessment criteria.

\section{References}

Aoki, Nobuo, Jiahao Zhang, and Subin Xu. 2016. "Research on 'The International Academic Conference on Chinese Industrial Architecture Heritage (2008-2014)'” Architecture \& Culture 2016 (4): 100-103.

Aoki Nobuo, Subin Xu, and Ruoran Wang. 2016. "Discussion about the Human Value Orientation-The Negative Value of Modern Industrial Heritage Cognition and Cultural Strategy." Urban Environment Design 102(B): 196-203.

Chen, Jiamin, and Subin Xu. 2017. “The Economic Value Assessment of Cultural Capital of Industrial Heritage after Renovation-A Case Study of '798' Art Zone.” Paper presented at the International Conference on East Asian Architecture, Tianjin, October 13-17.

Du, Xin. 2013. "Mapping of Industrial Architectural Heritage Based on BIM." Master degree thesis, Tianjin University.

Fairbank Centre for Chinese Studies, the Institute for Chinese Historical Geography at Fudan University. CHGIS [DB/OL], Cambridge: Harvard University, 2012 [201611-24]. https://www.fas.harvard.edu/ chgis/data/ chgis/v5/

Feng, Yuchan. 2016. "Research on the Reuse of Chinese Modern Industrial Heritage from the Aspect of Authenticity." Master degree thesis, Tianjin University.

Hao, Shuai, Shan Xue, Shuangchen Chen, and Xin Du. 2012. "Case Study about the Reconstruction of Industrial Heritage Sites in Aisa." In Proceedings of the Third Chinese Academic Forum of Industrial Heritage 2012.

He, Jiangfeng. 2009. "Shiyi changji yiwei shi-yi Tianjin jiqiju de chaoxian xuetu wei gean yanjiu." [Case Study of the Korean Handicraft Apprentices in Tianjin Machinery Bureau] Researches in Chinese Economic History 2009 (4): 147-154.

Ji, Hong, Subin Xu, and Nobuo Aoki. 2011. "Yangshi Lei yu tianjin jindai gongye jianzhu: yi haiguangsi xinggong yu jiqiju weili." [Yangshi Lei and the Modern Industrial Buildings of Tianjin: Case Study on the Imperial Palaces for the Temporary Dwelling and the Machine Bureau of Haiguang Temple] Architectural Journal 2011 (s1): 93-97.

Lai, Shixian. 2016. "Guanyu zhongguo jindai chengshi gongye fazhan lishi fenqi wenti de yanjiu." [A Research on the Developments of the Technology of Chinese Early Modern Industrial Buildings] Industrial Construction (forthcoming).

Lai, Shixian, Subin Xu. 2016. "Research on the Development of Brick-making Technology in Modern China." 
History of Architecture 2016 (2): 201-212.

Lai, Shixian, Subin Xu. 2017. "Zhongguo jindai zhuanwa biaoshi yanjiu." [Research on Icons on Brick and Tile in Modern China] Architecture \& Culture 2017 (6): 48-50.

Yu, Changjiang, Kun Qian, and Haiqing Li. "Zhongguo huofuman yaojianzao moshi de diaocha yu huanjingshiyingxing fenxi." [Building Mode Survey and Environmental-adaptability Analysis of Hoffmann Kiln in China] Heritage Architecture 2 (1): 106-113.

Lai, Shixian. 2016. Research on the Construction Technology of Chinese Modern Industrial Buildings with the Perspective of Architectural Materials (1840-1949).

Li, Songsong, Subin Xu, Nobuo Aoki. 2016. "Gongyeyichan wenwubaohu guihua wenti tanxi." [Analysis on Protection Planning of Industrial Heritage Relics] Traditional Architecture 2016: 114-121.

Li, Xin, Lian Hu, Lin Wang, and Subin Xu. 2015. "Gongyeyichan gaizao gengxin de yingxiangyinsu de fenxi: yi Beijing Tianjin Shanghai Suzhou weili”. [Analysis of main Factors Influencing on Industrial Heritage's Reuse-Cases Study on the Textile Industrial Heritage in Beijing, Tianjin Shanghai and Suzhou] Architecturees Culture 2015 (6): 96-100.

Liu, Jing. 2017. "Zhongguo jindai chengshi shikong yanbian fenxi." [Temporal-Spatial evolution of Modern urban industry in China] City Planning Review (forthcoming).

Liu, Yu. 2016. "Analysis on the Evolution of Time and Space of the Urban Industry in Modern China." PhD. diss., Tianjin University.

“Constitution of the People's Republic of China." 1988. Paragraph IV of Article 10..

Qiu, Shuang, Jin Zuo, and Jingtao Huang. 2014. "Heyue shijiao xiade chanye yicun zailiyong guihua moshi yanjiu: yi Tianjin mianfangsanchang weili." [Study on Industrial Heritage Reuse Planning Modes from the Perspective of Contract: A Case Study of Tianjin Third Cotton Textile Factory] Urban Development Studies 2014 (3): 112-118.

Shi, Yue. 2014. Application of BIM in information collection and management of industrial heritage. Tianjin University, 2014.

Sun, Yuejie, Subin Xu, Nobuo Aoki. 2015. "Studies and Reuse Design on Luoyang Jianxi Residential Neighbourhood of 1950's." Urban Studies 22 (1): 51-55.

Throsby, David. 2010. The Economics of Cultural Policy. Cambridge: Cambridge University Press.

Throsby, David. 2001. Economics and Culture. Cambridge:
Cambridge University Press.

Xu, Subin, and Nobuo Aoki. 2017. “Zaikao Zhongguo gongye yichan jiazhi de kuangjia." [Further Research of the Framework of Chinese Industrial Heritages] In Collected Papers of the Eighth International Academic Conference on Chinese Industrial Heritage Nanjing 2017.

$\mathrm{Xu}$, Subin, and Nobuo Aoki. 2012. "Guanyu gongye yichan wanzhengxing de sikao." [Considerations the Integrity of Industrial Heritages] In Proceedings of the Third Chinese Academic Forum of Industrial Heritage 2012. Harbin Institute of Technology, November.

Xu, Subin, Shuai Hao, and Nobuo Aoki. 2016. "Kailuan meikuang gongye yichanqun yanjiu jiqi jiazhirending de tantao." [A Study on the Industrial Heritage Group of Kailuan Mining Area and Its Value Assessment] New Architecture 2016 (3): 10-13.

Xu, Subin. 2014. "20 shijichu kaibuchengshi Tianjin de riben shourong: yi kaogongchang (shangping chenliesuo) ji quanyehuichang weili." [The Influence of Japan in Tianjin, the Port-opening City, in the Early 20 Century-Cases Study of the Commercial Museum and Quanye Bazaar] Urban History Research 2014 (1): 188-203.

$\mathrm{Xu}$, Subin, Nobuo Aoki. 2018. "Zhongguo gongye yichan jiazhi kuangjia sikao.” [Reflections on the Value Framework of Chinese Industrial Heritages] Industry and Technology History Research 3 (3) (forthcoming).

Xu, Subin, Nobuo Aoki. 2016. "Cunliangguihua shidai de gongyeyichan baohu." [Industrial Heritage Protection in the Period of Inventory Planning] Journal of Landscape Research 2016 (6): 77-82.

$\mathrm{Xu}$, Subin. 2018. “The Spread of Industrial Technology of Modern Europe and Asia from the Railway Technicians in Late Qing Dynasty: Case Study of British and Japanese Technicians." Urbanism and Architecture 2018 (3) (forthcoming).

Xu, Subin, Shixian Lai, Jing Liu, and Nobuo Aoki. 2017. "Guanyu zhongguo jindai chengshi gongyefazhan lishi fenqiwenti de yanjiu". [Research on Historical Stages of Urban Industrial Development in China] The Architect 2017 (12): 40-47.

Xu, Subin, Fei Peng, and Xu Zhang. 2015. "Changshi tudizhengce dui gongyeyichanbaohu yu zailiyong de yingxiangfenxi." [Analysis of the Impact of Urban Land Policy on the Protection and Reuse of Industrial Heritage] Journal of Tianjin University (Social Sciences) 2015 (5): 385-390.

$\mathrm{Xu}$, Subin, Jiahao Zhang, Nobuo Aoki, and Cong Wu. 
2015. “Zhongdianchengshi gongyeyichan GIS shujuku jiangouyanjiu: yi Tingjin weili." [Construction of GIS Database for Industrial Heritage in Key Cities: Taking Tianjin as an Example] Industrial Construction 2015(8).

Xu, Subin, Dandan Zhong, and Kuolei Hu. 2016. "Qingdao gongyeyichan baohu zailiyong moshi de xuanzejizhi yanjiu." [The Selection Mechanism of Conservation Reuse Mode of Industrial Heritage Qingdao] Industrial Construction 2016 (2): 18-22.

$\mathrm{Xu}$, Subin, and Dandan Zhong. 2015. "Gaoxiao daidongxia de wenhuachanye yu gongyeyichan zailiyong xietongfazhan". [The Analyses on Composition of Luoyang Industrial Heritage in 1950's] Urban Development Studies 2015 (11): 14-19.

$\mathrm{Xu}$, Subin, Yuejie Sun, and Nobuo Aoki. 2015. "Luoyang 'yiwu' shiqi sushizhuzhai jiefang kaocha yu gaizao tansuo". [The Analyses on Composition of Luoyang Industrial Heritage in 1950s] Urban Development Studies 2015 (8): 112-117.

$\mathrm{Xu}$, Subin, and Tian Li. 2015. "Instruments of Domination and Cultural Symbols- Study on the Eight Barracks of Tianjin Concession”. Architecture \& Culture 2015 (3): 105-106.

Yan, Mi, Nobuo Aoki, and Subin Xu. 2015. "Grading Protection of Industrial Heritage of Tianjin Soda Plant Based on Value Evaluation." Industrial Construction 2015 (5): 34-37.

Yu, Lei, Nobuo Aoki, and Subin Xu. 2016. "Jindai Zhongguo zhonggongye gongye yichan jiazhi pingjia yanjiu." [Value Evaluation of Heavy Industrial Heritages of China.] In Proceedings of the Sixth Chinese Academic Forum of Industrial Heritage 2015, edited by Wenyi Zhu and Boying Liu, 50-59. Beijing: Tsinghua University Press.

Yu, Lei, Nobuo Aoki, and Subin Xu. 2017. "Gongyeyichan jiazhi pingjia fangfa yanjiu." [Study on the Value Evaluation Methodology of the Industrial Heritage] Chinese Cultural Heritage 2017 (1): 59-64.

Yu, Lei, Nobuo Aoki, and Subin Xu. 2016. "Ying mei jia sanguo gongyeyichan jiazhi pingding yanjiu”. [A Study on the Appraisal of Industrial Heritage in the UK, the US and Canada] Architectural Journal 2016 (2): 1-4.

Zhang, Jiahao, Xu, Subin, and Nobuo Aoki. 2016. "GIS zai Beiyang Dagu chuanwu baohuguihua qianqizhong de yingyong." [Application of GIS in the Early Stage of Conservation Planning for North Navy's Taku Dockyard.] In Proceedings of the Seventh Chinese Academic Forum of Industrial Heritage 2016, 670-675. College of
Architecture and Urban Planning, Tongji University.

Zhong, Dandan. 2016. "The Research of the Motivation of the Integration Development of Industrial Heritage Conservation Reuse and Cultural Industrial in China." PhD diss., Tianjin University.

Zhong, Dandan, Subin Xu, Lin Wang, and Lian Hu. 2016. "Huabo tudishiyongquan zhidu yingxiangxiade gongyeyichan baohu zailiyong: yi Beijing Shanghai weili." [Preservation and Regeneration of Industrial Heritage with the Regulative Influence of Appropriation of Land-Use Rights: Case Studies in Beijing and Shanghai] Architectural Journal 2016 (3): 24-28.

Zhang, Yuqi, Lian Hu, Lin Wang, and Subin Xu. 2015. "Zhengcedaoxiang dui gongyeyichan zailiyong fangshi de yingxiang yanjiu." [Research on the Policy-oriented Influence to the Reconstruction Methods of Industrial Heritage] Architecture \& Culture 2015 (5): 98-101. 